References and Notes

1. Z. Xi, C. C. H. Khoo, S. L. Dobson, Science 310, 326 (2005).

2. A. A. Hoffmann et al., Nature 476, 454 (2011).

3. G. Bian, Y. Xu, P. Lu, Y. Xie, Z. Xi, PLoS Pathog. 6, e1000833 (2010).

4. L. A. Moreira et al., Cell 139, 1268 (2009).

5. Z. Kambris et al., PLoS Pathog. 6, e1001143 (2010).

6. G. L. Hughes, R. Koga, P. Xue, T. Fukatsu, J. L. Rasgon, PLoS Pathog. 7, e1002043 (2011).

7. C. L. Brelsfoard, Y. Séchan, S. L. Dobson, PLoS Negl. Trop. Dis. 2, e129 (2008).

8. P. Kittayapong, K. J. Baisley, V. Baimai, S. L. O'Neill, J. Med. Entomol. 37, 340 (2000)

9. Materials and methods are available as supplementary materials on Science Online.

10. S. L. Dobson, E. J. Marsland, W. Rattanadechakul, Genetics 160, 1087 (2002).

11. U. Tram, P. M. Ferree, W. Sullivan, Microbes Infect. 5, 999 (2003).

12. Z. Xi, ]. L. Dean, C. Khoo, S. L. Dobson, Insect Biochem. Mol. Biol. 35, 903 (2005).
13. P. Lu, G. Bian, X. Pan, Z. Xi, PLoS Negl. Trop. Dis. 6 e1754 (2012).

14. X. Pan et al., Proc. Natl. Acad. Sci. U.S.A. 109, E23 (2012).

15. C. M. Cirimotich et al., Science 332, 855 (2011).

16. S. Kumar et al., Proc. Natl. Acad. Sci. U.S.A. 100, 14139 (2003).

17. G. L. Hughes, A. D. Pike, P. Xue, J. L. Rasgon, PLoS ONE 7, e36277 (2012)

18. C. J. McMeniman, G. L. Hughes, S. L. O'Neill, J. Med. Entomol. 48, 76 (2011).

19. S. I. Hay et al., PLoS Med. 7, e1000209 (2010).

20. M. E. Sinka et al., Parasit Vectors 4, 89 (2011).

21. The malERA Consultative Group on Vector Control, PLoS Med. 8, e1000401 (2011).

22. R. E. Sinden, Y. Alavi, J. D. Raine, Insect Biochem. Mol. Biol. 34, 625 (2004).

23. Y. Dong et al., PLoS Pathog. 7, e1002458 (2011).

Acknowledgments: This work was supported by NIH grants R01Al080597, R21AI082141, and R01AI061576 and a grant from the Foundation for the NIH through the Grand Challenges in Global Health Initiative of the Bill and Melinda Gates Foundation. We are grateful to the Johns Hopkins Malaria Research Institute Parasitology and Insectary Core Facilities and thank S. O'Neill, A. A. Hoffmann, and S. L. Dobson for their comments and suggestions and D. McClellan and S. Thiem for editorial assistance. Z.X. is also affiliated with Guangzhou WolbaKi Biotech Co., LTD. Data for this report are archived as supplementary materials on Science Online.

\section{Supplementary Materials}

www.sciencemag.org/cgi/content/ful//340/6133/748/DC1

Materials and Methods

Figs. $\mathrm{S} 1$ to $\mathrm{S3}$

Table $\mathrm{S1}$

References (24-30)

6 February 2013; accepted 15 March 2013

$10.1126 /$ science. 1236192

\title{
Delineating Antibody Recognition in Polyclonal Sera from Patterns of HIV-1 Isolate Neutralization
}

Ivelin S. Georgiev, ${ }^{1 *}$ Nicole A. Doria-Rose, ${ }^{1 *}$ Tongqing Zhou, ${ }^{1 *}$ Young Do Kwon, ${ }^{1 *}$ Ryan P. Staupe, ${ }^{1}$ Stephanie Moquin, ${ }^{1}$ Gwo-Yu Chuang, ${ }_{1}^{1}$ Mark K. Louder, ${ }^{1}$ Stephen D. Schmidt, ${ }^{1}$ Han R. Altae-Tran, ${ }^{1}$ Robert T. Bailer, ${ }^{1}$ Krisha McKee, ${ }^{1}$ Martha Nason, ${ }^{1}$ Sijy $0^{\prime}$ Dell, ${ }^{1}$ Gilad Ofek, ${ }^{1}$ Marie Pancera, ${ }^{1}$ Sanjay Srivatsan, ${ }^{1}$ Lawrence Shapiro, ${ }^{1,2}$ Mark Connors, ${ }^{3}$ Stephen A. Migueles, ${ }^{3}$ Lynn Morris, ${ }^{4,5,6}$ Yoshiaki Nishimura, ${ }^{7}$ Malcolm A. Martin, ${ }^{7}$ John R. Mascola, ${ }^{1} \dagger$ Peter D. Kwong ${ }^{1} \dagger$

Serum characterization and antibody isolation are transforming our understanding of the humoral immune response to viral infection. Here, we show that epitope specificities of HIV-1-neutralizing antibodies in serum can be elucidated from the serum pattern of neutralization against a diverse panel of HIV-1 isolates. We determined "neutralization fingerprints" for 30 neutralizing antibodies on a panel of 34 diverse HIV-1 strains and showed that similarity in neutralization fingerprint correlated with similarity in epitope. We used these fingerprints to delineate specificities of polyclonal sera from 24 HIV-1-infected donors and a chimeric siman-human immunodeficiency virus-infected macaque. Delineated specificities matched published specificities and were further confirmed by antibody isolation for two sera. Patterns of virus-isolate neutralization can thus afford a detailed epitope-specific understanding of neutralizing-antibody responses to viral infection.

$\mathrm{U}$ pon infection or vaccination, the adaptive immune system typically generates polyclonal antibody responses that recognize multiple epitopes (1-3). The serologic characterization of such polyclonal responses can inform vaccine design by elucidating which epi-

${ }^{1}$ Vaccine Research Center, National Institute of Allergy and Infectious Diseases, National Institutes of Health, Bethesda, MD 20892, USA. ²Department of Biochemistry and Molecular Biophysics, Columbia University, New York, NY 10032, USA ${ }^{3}$ Laboratory of Immunoregulation, National Institute of Allergy and Infectious Diseases, National Institutes of Health, Bethesda, MD 20892, USA. ${ }^{4}$ National Institute for Communicable Diseases, Sandringham 2131, South Africa. ${ }^{5}$ University of the Witwatersrand, Johannesburg 2001, South Africa. ${ }^{6}$ Centre for the AIDS Programme of Research in South Africa (CAPRISA), Durban 4013, South Africa. ' ${ }^{7}$ Laboratory of Molecular Microbiology, National Institute of Allergy and Infectious Diseases, National Institutes of Health, Bethesda, MD 20892, USA.

*These authors contributed equally to this work. †Corresponding author. E-mail: jmascola@nih.gov (].R.M.); pdkwong@nih.gov (P.D.K.) topes on the antigen are immunodominant and/or targets of pathogen-specific neutralizing antibodies. Such serologic analysis can further lead to the isolation of new monoclonal antibodies that may be of therapeutic value. As a result of extensive effort to understand the antibody response to viral infection, recent years have seen a surge in the isolation of monoclonal antibodies against HIV-1, influenza, hepatitis C, and other viruses (4-15). The link between polyclonal sera and component monoclonal antibodies, however, remains complex and difficult to decipher, in part, because of the extraordinary diversity of circulating antibodies. Viral genetic diversity can be an integral mechanism of immune evasion (16-22); this same diversity may, however, also provide a means by which to understand antibody responses $(23,24)$. Specifically, monoclonal antibodies targeting the same epitope on an antigen are likely to be affected in a similar way by diversity in that epitope region. When presented with a diverse set of viral isolates, monoclonal antibodies may thus exhibit characteristic neutralization patterns or "neutralization fingerprints" (Fig. 1). Furthermore, neutralization patterns of a polyclonal serum could be viewed as the combined effect of the neutralization fingerprints of component monoclonal antibodies, and, if this relationship could be deconvoluted, then serum neutralization would serve as a predictor of componentantibody specificity.

To test this conjecture, we selected HIV-1 because of its high viral sequence diversity, the availability of well-characterized sera and antibodies, and the limited number of sites of vulnerability targeted by neutralizing antibodies on the HIV-1 spike (Env). These sites encompass the CD4binding site (CD4bs), a variable loop V1/V2 site, and a glycan-V3 site on glycoprotein gp120, and the membrane-proximal external region (MPER) on gp41 (4-7, 13, 14, 25-35). The same site of vulnerability may encompass multiple epitopes and, as a result, can be targeted by antibodies with diverse specificities. To determine whether the neutralization fingerprints of HIV-1 monoclonal antibodies are a reflection of their epitope specificities, we utilized neutralization data for a panel of 34 diverse HIV-1 isolates (table S1), for 30 monoclonal antibodies recognizing diverse epitopes on HIV-1 Env, and for two variants of the CD4 receptor (table S2). Neutralization fingerprints for antibodies known to target similar epitopes correlated significantly better (Spearman correlation) than fingerprints of antibodies targeting different epitopes (fig. S1). On the basis of the neutralization-correlation values, antibodies were grouped into 10 clusters (Fig. 2A) (36), by using a clustering cutoff chosen to agree with known antibody structures and epitope-mapping (4-6, 13-15, 25-27, 37-40, 41). Two antibodies, $8 \mathrm{ANC} 195$ and HJ16, whose precise epitopes are currently unknown, clustered separately $(5,15)$, whereas all of the other antibody clusters could be mapped to known sites of Env vulnerability.

Overall, neutralization fingerprints appeared to exhibit sufficient specificity to successfully distinguish between antibodies targeting different 
epitopes on the same overall site of vulnerability (42). The MPER site was recognized by two antibody clusters, each consisting of two antibodies (2F5/m66.6 and 10E8/4E10); this agreed with known MPER-antibody epitopes (fig. S2) and demonstrated the dominant contribution of the recognized epitope, rather than particulars of antibody orientation, to the antibody-neutralization fingerprints: 10E8 and 4E10 recognize the same epitope but use substantially different antibody heavy- and light-chain orientations $(13,43)$. The $\mathrm{V} 1 / \mathrm{V} 2$ site was recognized by a single cluster of PG9-like antibodies. The glycan-V3 site was recognized by two clusters of antibodies: PGT128like antibodies that target a glycopeptide-epitope and antibody $2 \mathrm{G} 12$ that targets a cluster of glycans including N332 (44) on the gp120 surface $(25,26,45)$. Last, the CD4bs was targeted by at least three clusters, including those of antibody b12, CD4, and the VRC01-like antibodies $(27,38)$.

VRC01-like antibodies represented the largest fraction in the analyzed data set and formed the largest antibody-neutralization cluster; they could be grouped around VRC01, a prototypical broad and potent CD4bs antibody capable of neutralizing $90 \%$ of circulating HIV-1 strains $(4,27)$. The VRC01-like cluster included antibodies known to target similar epitopes on gp120, as confirmed by antibody structures in complex with gp 120 (VRC01, VRC03, VRC-PG04, and NIH45-46) and epitope-mapping experiments $(4,5,14,27,40)$. Although published epitope maps suggest that antibody VRC06 recognizes an epitope composed of both CD4- and co-receptor-binding sites (46), the cocrystal structure of VRC06 in complex with core gp120 revealed a similar mode of gp120 recognition by VRC06, as compared with both VRC01 and VRC03 (Fig. 2B), consistent with the similarity observed for the neutraliza- tion fingerprints of these antibodies (47). These results indicate that clustering of antibodies based on neutralization fingerprints can be an accurate delineator of antibody-epitope specificity.

To assess whether the neutralization signal from polyclonal sera could be deconvoluted into component-antibody specificities, we analyzed neutralization data for sera from HIV-infected donors (48). The neutralization fingerprints for all antibodies within a given cluster were used to create a single representative neutralization fingerprint for the cluster, resulting in a reference set of 10 epitope-specific neutralization fingerprints, one for each antibody cluster (fig. S3) (41). The neutralization pattern for each serum was taken to be a linear combination of the reference-set fingerprints, resulting in an estimate of the relative contribution to serum neutralization (i.e., neutralization prevalence) of the respective componentantibody specificities as described in (41) and Fig. 1.

For most sera, multiple specificities were predicted (Fig. 3) - as might be expected from the polyclonal nature of sera $(1-3)$. To evaluate the neutralization-based serum-epitope predictions, we compared them with component-antibody epitopes determined by other experimental means. We first compared predictions for which serumneutralization specificities were already known through antibody isolation or extensive epitope mapping. The neutralization-based prediction for donor 45, the source of VRC01 and other VRC01like antibodies (4), indicated a strong signal for VRC01-like antibodies (Fig. 3A) (49). The prediction for donor N152, the source of antibody 10E8 (13), showed a dominant signal for the 10E8-like cluster (Fig. 3A). Furthermore, longitudinally sampled sera from donor CAP256 showed a PG9-like signal at each of four time points, both by extensive experimental epitope mapping (50) and computational predictions
(Fig. 3B). Last, sera from a chimeric simian-human immunodeficiency virus (SHIV)-infected macaque showed a dominant PGT128-like signal at each of four time points (Fig. 3C), in agreement with published epitope-mapping experiments (51). The neutralization-based method for delineating component-antibody epitopes was thus successful in identifying major antibody responses for sera with confirmed specificities.

Next, we applied our method to prospectively delineate specificities for sera from $21 \mathrm{HIV}-1-$ infected donors (52) and compared the results to (standard) experimental serum mapping data (Fig. 3D): Published assays were used to map serum antibody specificity toward the CD4bs, V1/V2, glycan-V3, and MPER regions $(41,53)$. The degree of correspondence between the neutralizationbased prediction method and standard mapping varied for different sera, with discrepancies partly attributable to known limitations in current standard mapping assays (54) (table S4). Nonetheless, the agreement between the two methods was reasonably good, with at least one of the top two neutralization-delineated specificities identified by standard mapping in $\sim 85 \%$ of the tested sera (Fig. 3E) (55).

To further validate our predictions, we selected two sera with different predicted specificities and prevalence levels: (i) serum from donor $127 / \mathrm{C}$ predicted to have a dominant VRC01-like specificity with a prevalence level of 0.60 and (ii) serum from donor $\mathrm{N} 27$ predicted to have a PGT128-like specificity with a prevalence level of 0.33 (Fig. 3D). We cloned CD4bs antibodies from donor $127 / \mathrm{C}$ peripheral blood mononuclear cells (PBMCs) by using an antigen-specific protein probe (RSC3), as described previously (4). We identified two somatic variants, VRC23 and VRC23b that showed strong similarity to other VRC01-like antibodies by germline-gene usage,

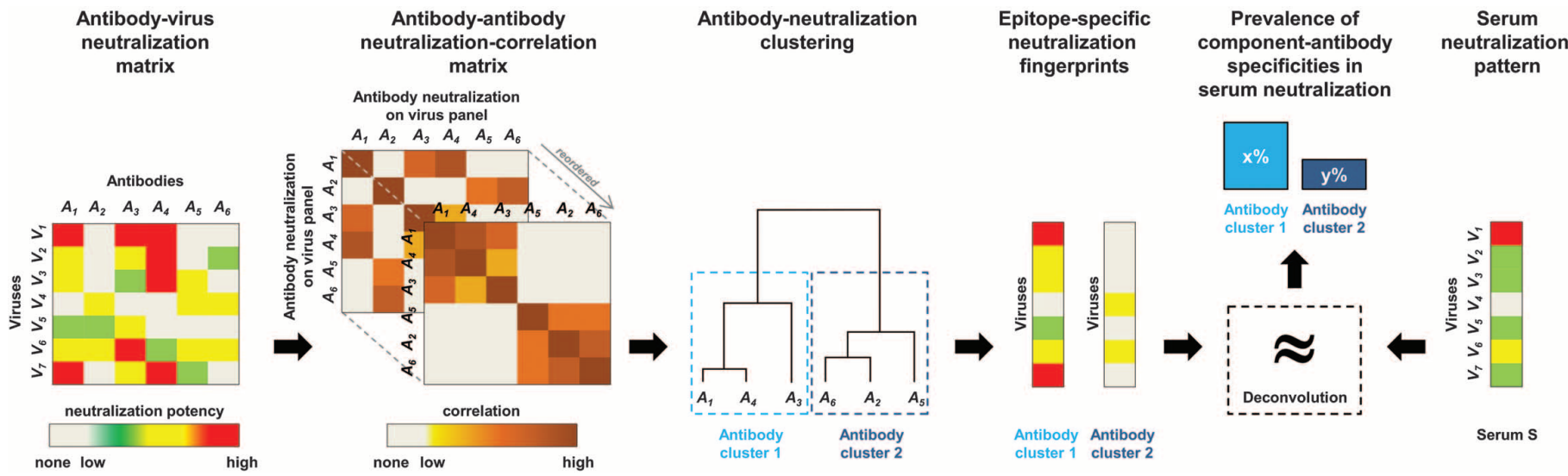

Fig. 1. Definition of antibody neutralization fingerprints and determination of antibody specificities from serum patterns of neutralization. The neutralization fingerprint for an antibody on a panel of diverse viruses may contain sufficient information to define both antibody epitope and molecular specificities of recognition. Here, we show how information from a neutralization matrix can be transformed to delineate epitope specificity. First, a database of neutralization fingerprints for known antibodies can be constructed on the basis of clustering of antibody-neutralization behavior: The neutralization fingerprints for antibodies (columns: $A_{1}$ to $A_{6}$ ) are shown in a neutralization matrix [first (leftmost) panel], with colors representing neutralization potencies against specific viruses (rows: $V_{1}$ to $V_{7}$ ); the correlations between antibody neutralization fingerprints (second panel) can help form epitope-specific antibody clusters (third panel). The resultant epitope-specific neutralization fingerprints (fourth panel) can be used to interrogate patterns of neutralization from polyclonal sera. Deconvolution of the serum-neutralization pattern (last panel) into epitope-specific fingerprints identifies epitope specificity of the component antibodies (fifth panel). 
sequence signature, binding characteristics, and neutralization (Fig. 4A and figs. S4 to S7) (41). Furthermore, the crystal structure of VRC23 in complex with gp120 revealed substantial similarities with the mode of gp120 recognition by VRC01 and other VRC01-like antibodies (56), confirming the neutralization-based prediction of VRC01-like antibody specificity in serum 127/C. By comparison, standard experimental serum mapping showed borderline evidence for CD4bs activity, with RSC3-based inhibition of $127 / \mathrm{C}$ neutralization reaching only $20 \%$ (fig. S11). For donor N27, standard serum mapping (Fig. 3D) indicated the presence of glycan-reactive antibodies. We used single B cell culture $(5,13,41)$ and recovered one neutralizing antibody, VRC24 (fig. S4). Binding and neutralization assays for VRC24 suggested a glycan-V3 epitope (figs. S12 to S14), with general similarity to (but also some differences from) other antibodies in the PGT128like cluster. Thus, for both donors $127 / \mathrm{C}$ and N27, we successfully confirmed the existence of antibodies with specificities predicted by the neutralization-based method. Furthermore, the neutralization fingerprints for antibodies VRC23 and VRC24 clustered appropriately - within the VRC01-like cluster for VRC23 and most closely to (though not fully intermingled with) the PGT128like cluster for VRC24 (Fig. 4B) - which demonstrated that, in addition to delineating serum specificity, neutralization fingerprints allow for prospective prediction of monoclonal antibody epitopes.
Analysis of sera from HIV-1-infected donors and, more recently, from SHIV-infected macaques is revolutionizing our understanding of the ability of the humoral immune system to recognize and to neutralize HIV-1 (29-34). Such analysis generally begins with an assessment of serum neutralization on small or moderately sized virus panels $(29,57)$ and then proceeds with epitope mapping and monoclonal antibody isolation. The ability to delineate componentantibody neutralization specificities directly from serum-neutralization data potentially transforms this process, by providing a computational shortcut for procedures of standard epitope mapping, monoclonal antibody isolation, and crystal structure determination of the epitope (58). Such a

A
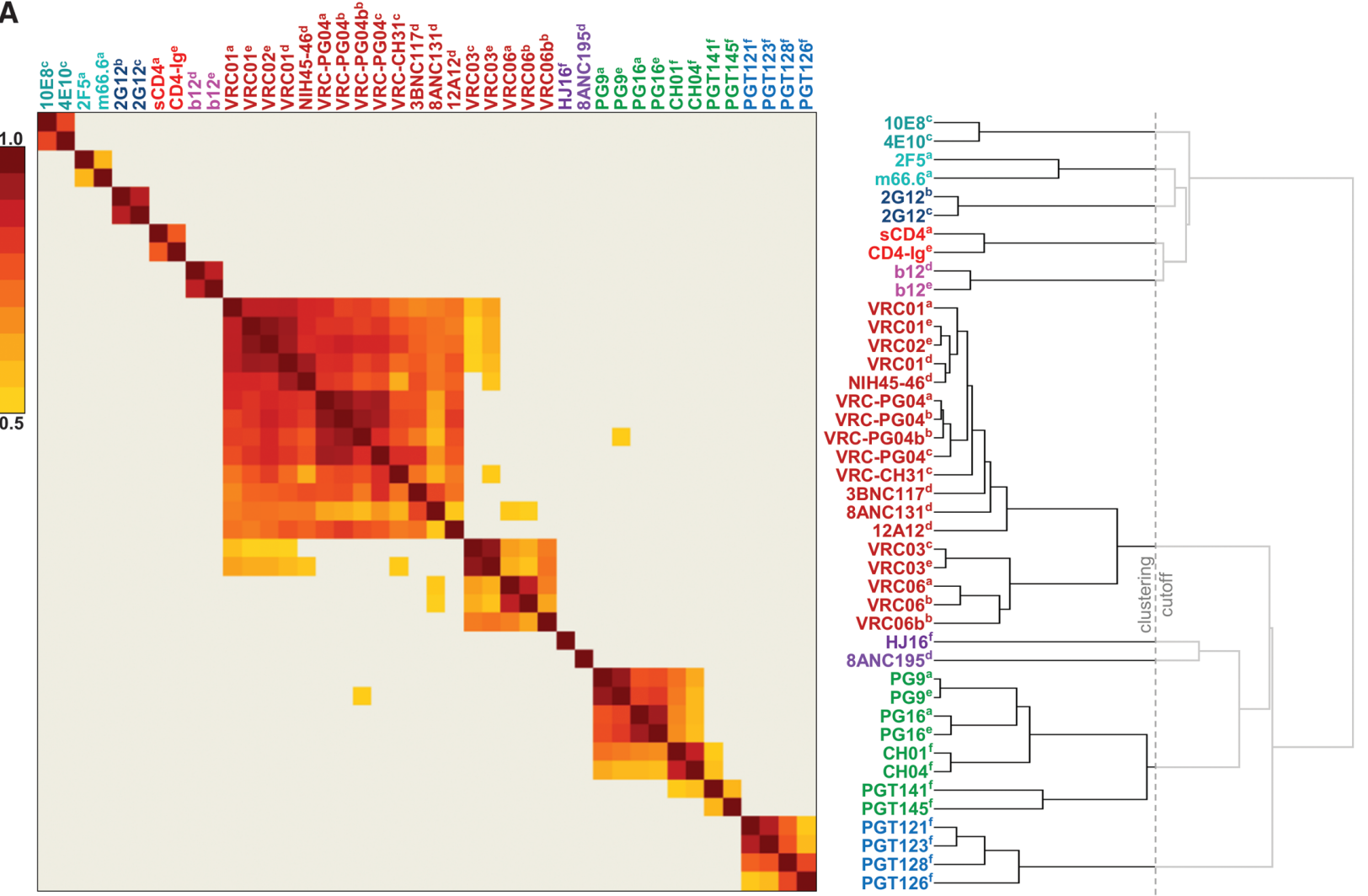

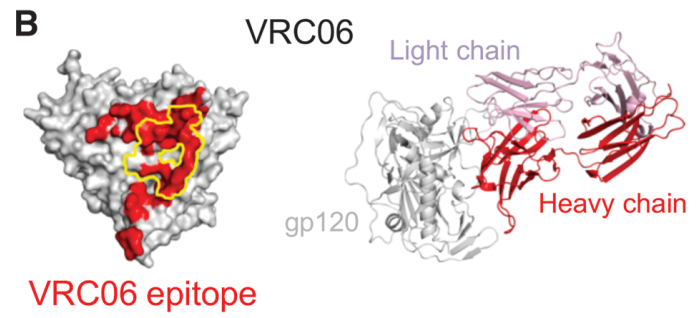

Fig. 2. Neutralization-based clustering of HIV-1 antibodies. (A) Antibody-antibody neutralization-correlation matrix. A set of HIV-1-neutralizing antibodies along with two variants of CD4 were assessed for neutralization on a panel of 34 diverse HIV-1 isolates. Input from six neutralization panels (tables S5 and S6) were used to define this correlation matrix and are indicated by superscripts a through $f$ next to each antibody name, with several antibodies tested on multiple panels. Correlation results are shown in heat-map representation from 0.5 (yellow) to 1.0 (dark brown) and were used as input for antibody clustering. The intersection

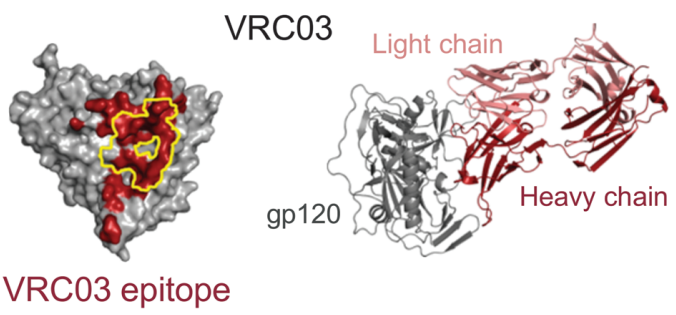

of the dotted vertical line with horizontal lines in the clustering tree defines the 10 antibody clusters: VRC01-like (maroon), PG9-like (green), PGT128-like (blue), 2F5-like (light blue), and 10E8-like (aqua), as well as separate clusters for b12, CD4, 2G12, HJ16, and 8ANC195. Antibody order was based on the clustering tree. (B) Comparison of gp120 recognition by antibodies VRC03 and VRC06, shown as epitope and 90 degree-rotated complex. Although VRC03 and VRC06 display substantial phenotypic differences (46), their neutralization fingerprints are highly correlated, and cocrystal structures reveal the same gp120 mode of recognition. 


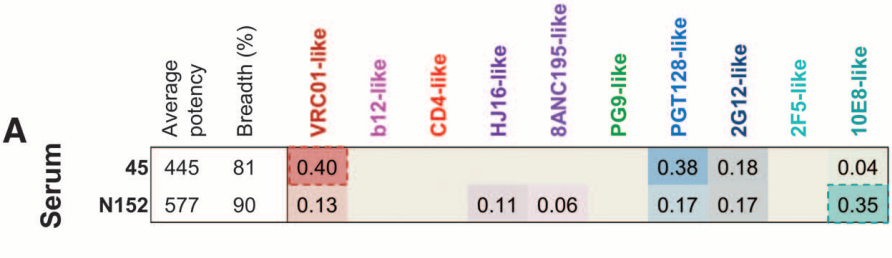

B

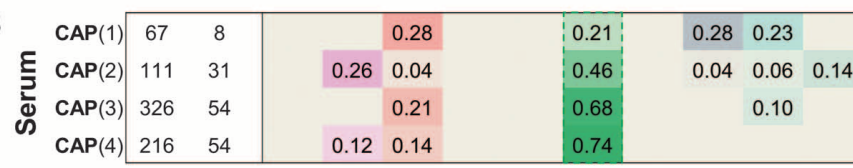

\begin{tabular}{|c|c|c|c|c|c|c|c|c|c|c|}
\hline CE8J(1) & 72 & 43 & 0.15 & 0.12 & 0.09 & & 0.52 & & 0.04 & 0.06 \\
\hline CE8J(2) & 127 & 57 & & 0.30 & & & 0.44 & 0.18 & & 0.06 \\
\hline E8J(3) & 161 & 62 & 0.02 & 0.22 & & & 0.57 & 0.08 & & 0.10 \\
\hline & 77 & 52 & 0.04 & 0.05 & & 1.08 & 0.58 & 14 & 0.10 & \\
\hline
\end{tabular}

Neutralization-based predictions

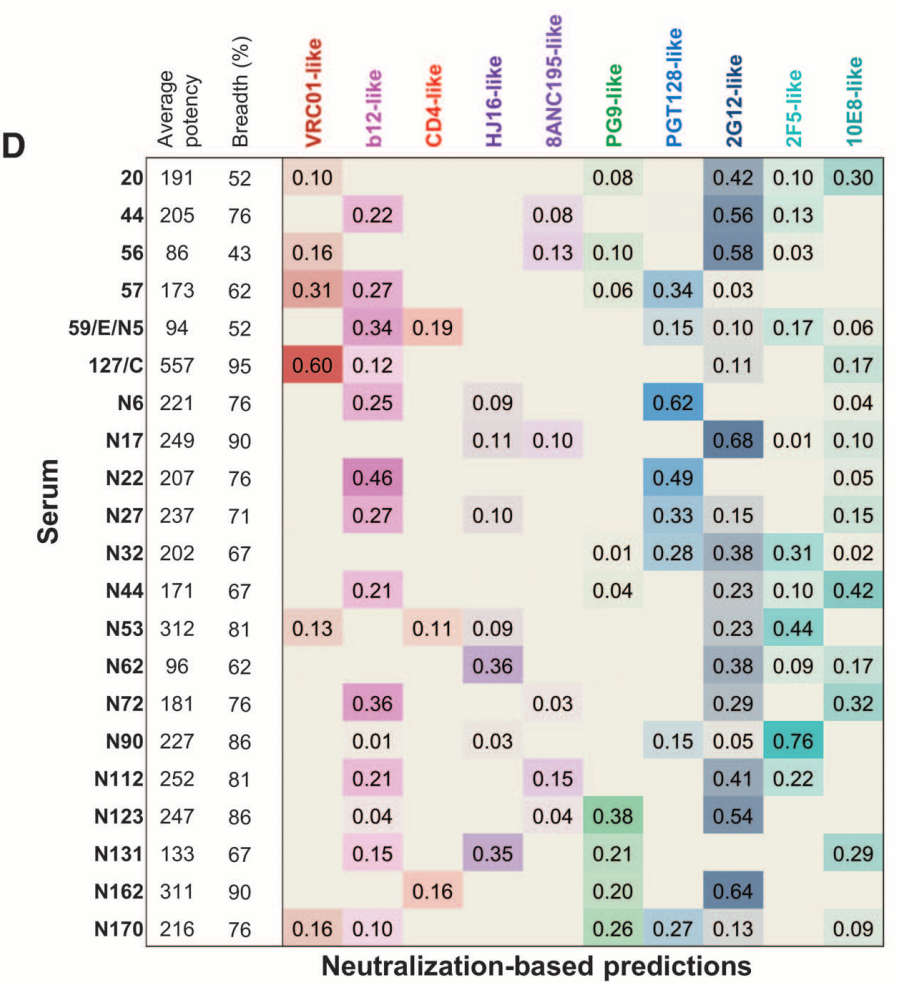

Fig. 3. Delineation of antibody specificities in HIV-1 sera with broadly neutralizing activity. For each serum, the predicted relative prevalence of the different reference-set antibody clusters (colored as in Fig. 2) is shown as a heat map, with darker intensity (higher fractional number) corresponding to a stronger neutralization signal (predicted prevalence level) by the respective antibody cluster. Numbers in each row add up to 1.00 , with numbers less than 0.01 not shown. Serum neutralization breadth is shown for the specific virus panels used, and average potency provided as geometric mean inhibitory dilution $\left(\mathrm{ID}_{50}\right)$. Predictions for $(\mathbf{A})$ serum from donor 45 (the source for antibody VRC01) and from donor N152 (the source for antibody 10E8), (B) sera from four different time points (years 1 to 4) postinfection from donor CAP256, and (C) sera from four different time points (weeks 40,
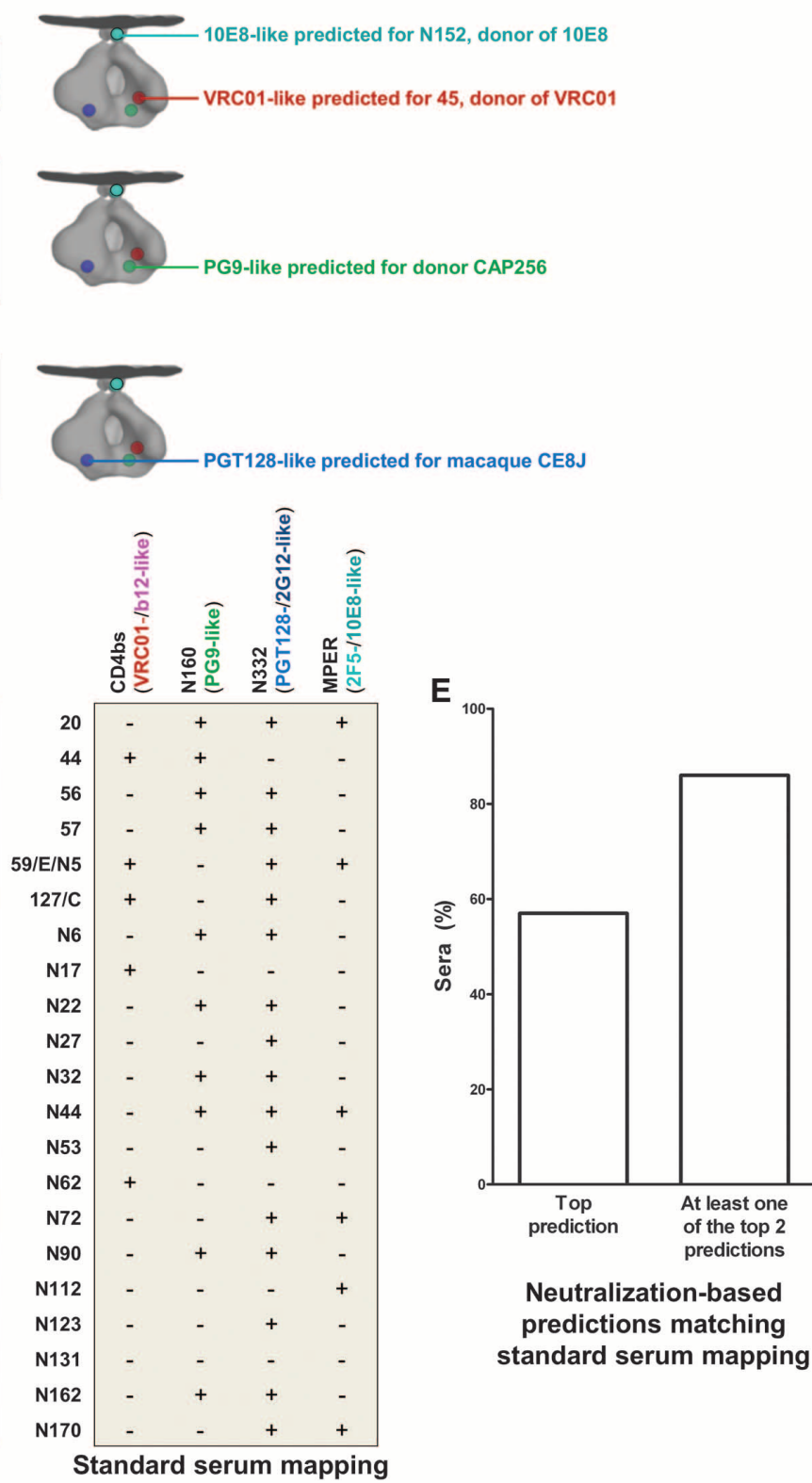

Neutralization-based predictions matching standard serum mapping

61,81 , and 100 ) postinfection from macaque CE8]. To the right of (A) to (C) are schematics of the putative locations on the HIV-1 Env trimer of the epitopes identified in the neutralization-based predictions and previously confirmed to be targeted by antibodies in the sera. (D) Neutralization-based predictions (left) and standard experimental mapping data (right) for an additional set of 21 sera (rows) and 10 epitopes (columns). Epitope groups (bold) for the experimental mapping data were defined on the basis of the assays used; the respective closely matching neutralization-based epitope clusters were grouped accordingly (shown in parentheses). Epitope groups are marked with a plus sign (+) if predicted by the mapping assays to be present in a given serum or with a "-" otherwise. (E) Concordance between neutralization-based and standard serum mapping.

short-cut should afford a more rapid and less arduous delineation of serum component-antibody epitopes and may be especially useful for studies in which sample volumes are limited. Two developments have been key to the success of the method and are likely to work synergistically to improve such neutralization-based delineation. First, application of robotic technology to neutralization assessment has made data from HIV-1-neutralization panels easier to obtain
$(14,57,59,60)$. Second, advances in monoclonal antibody identification have allowed for the recent isolation of many effective HIV-neutralizing antibodies, and structural analysis has delineated their sites of recognition $(20,35,61)$; although 
A A
analysis
VRC01-like

prediction

\begin{tabular}{l|lllll|}
\multirow{2}{*}{ Donor 127/C } & 0.60 & 0.12 & 0.11 & 0.17 \\
\cline { 2 - 4 }
\end{tabular}

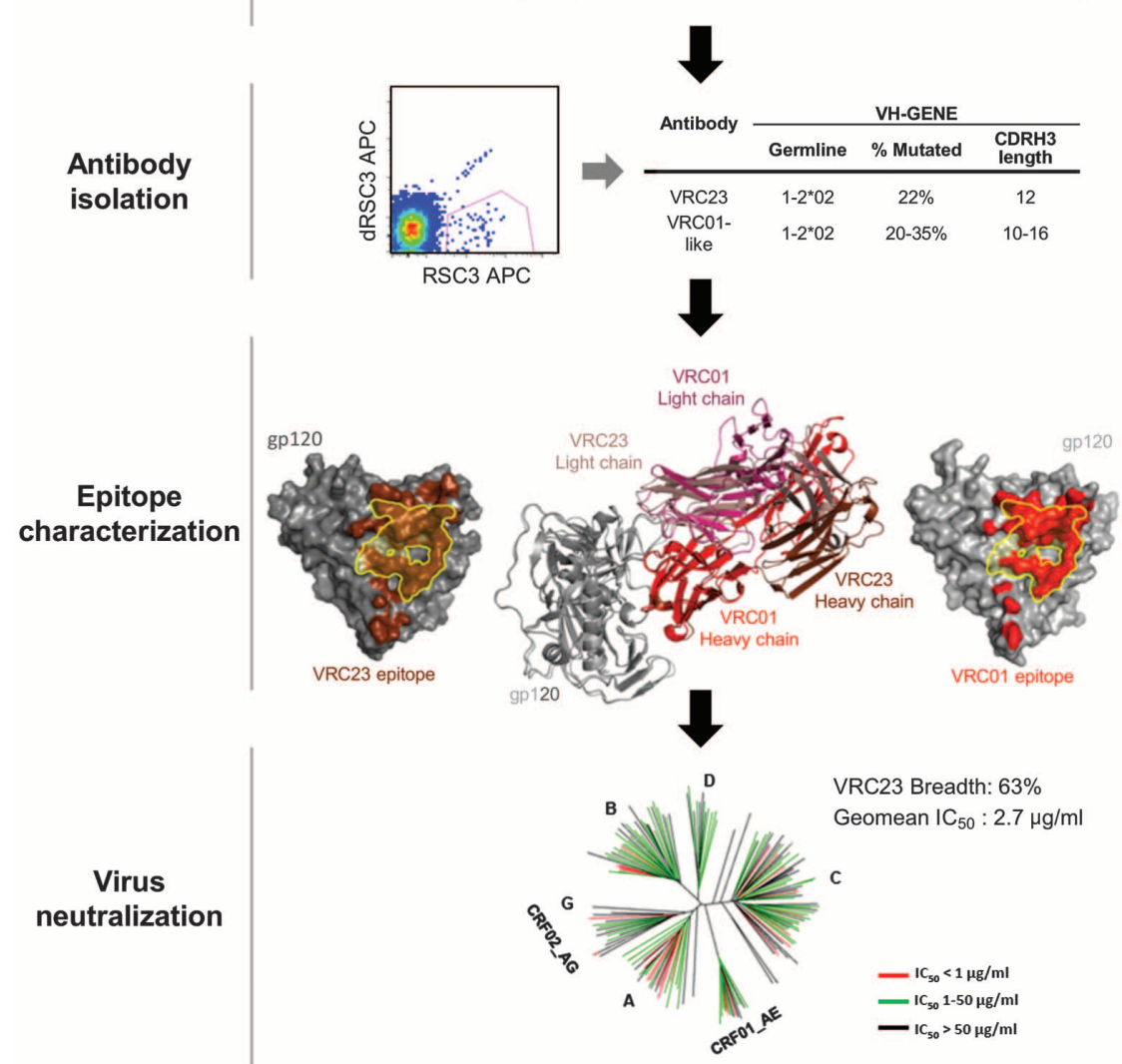

B

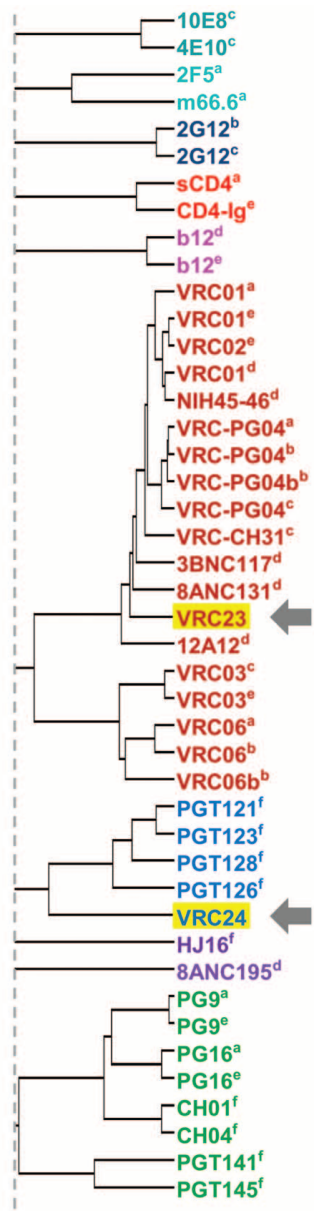

Fig. 4. Isolation and characterization of monoclonal antibodies isolated from sera identified by neutralization-based predictions. (A) Isolation and characterization of antibody VRC23 from donor 127/C. (Top row) Donor $127 / C$ was predicted to have VRC01-like antibodies. (Row 2) Immunoglobulin G-positive ( $\mathrm{gg}+$ ) B cells from donor $127 / \mathrm{C}$ were stained with the CD4bs-specific probe RSC3 and the CD4bs mutant dRSC3 and sorted. IgG variable genes were recovered by reverse transcription polymerase chain reaction, sequenced, and subcloned into heavy- and light-chain expression vectors. Like VRC01, the VRC23 heavy chain derives from the VH1-2*02 germline gene. The percent mutation from the germline $\mathrm{VH}$ gene is shown, as is the amino acid length (from the Kabat database) of the CDRH3 loop. (Row 3) VRC23-gp120 crystal structure shows gp120 recognition similar to that of VRC01. Polypeptide chains are depicted in ribbon representation (middle). The footprints of VRC23 (left, brown) and VRCO1 (right, red) on the surface of gp120 showed similar targeting of the CD4-defined initial site of vulnerability (yellow trace). (Bottom row) VRC23-neutralization dendrogram. VRC23 was tested against 178 genetically diverse Env-pseudoviruses representing the major HIV-1 clades. Neighbor-joining tree displays the protein distance of the respective gp160 sequences. Tree branches are colored by the neutralization potency of VRC23 against each particular isolate. The geometric mean $\mathrm{IC}_{50}$ of all neutralized viruses is shown. (B) Neutralizationbased antibody clustering shows VRC23 (highlighted) clustering with the VRC01-like antibodies and VRC24 (highlighted) clustering with the glycandependent PGT128-like antibodies, in agreement with epitope determination by atomic-level structure (VRC23) and mapping by mutagenesis (VRC23 and VRC24). currently identified sites are likely to dominate the neutralizing antibody response, highly effective antibodies against unknown and/or less-wellcharacterized epitopes continue to be identified $(53,62)$. Generally, epitope delineation based on neutralization fingerprints may provide a transformative strategy for screening sera or characterizing antibody specificities induced upon infection or vaccination against HIV-1 as well as other viruses.

\section{References and Notes}

1. J. Wrammert et al., Nature 453, 667 (2008).

2. J. F. Scheid et al., Nature 458, 636 (2009).

3. E. Traggiai et al., Nat. Med. 10, 871 (2004).

4. X. Wu et al., Science 329, 856 (2010).

5. J. F. Scheid et al., Science 333, 1633 (2011).

6. L. M. Walker et al., Nature 477, 466 (2011).

7. L. M. Walker et al., Science 326, 285 (2009).
8. M. Throsby et al., PLOS ONE 3, e3942 (2008).

9. ]. Sui et al., Nat. Struct. Mol. Biol. 16, 265 (2009).

10. B. Bartosch et al., Proc. Natl. Acad. Sci. U.S.A. 100, 14199 (2003)

11. ].-C. Meunier et al., J. Virol. 82, 966 (2008).

12. M. Perotti et al., J. Virol. 82, 1047 (2008).

13. J. Huang et al., Nature 491, 406 (2012).

14. X. Wu et al., Science 333, 1593 (2011).

15. D. Corti et al., PLOS ONE 5, e8805 (2010).

16. E. Delwart et al., J. Infect. Dis. 205, 875 (2012).

17. B. Korber, S. Gnanakaran, Current Opinion in HIV and AIDS 4, 408 (2009).

18. B. Korber et al., Br. Med. Bull. 58, 19 (2001).

19. P. D. Kwong, I. A. Wilson, Nat. Immunol. 10, 573 (2009).

20. D. R. Burton, P. Poignard, R. L. Stanfield, I. A. Wilson, Science 337, 183 (2012).

21. M. Bonsignori et al., Trends Microbiol. 20, 532 (2012).

22. G. B. Karlsson Hedestam et al., Nat. Rev. Microbiol. 6, 143 (2008).

23. Z. Cai, T. Zhang, X.-F. Wan, PLOS Comput. Biol. 6, e1000949 (2010)
24. D. J. Smith et al., Science 305, 371 (2004).

25. R. Pejchal et al., Science 334, 1097 (2011).

26. ]. S. McLellan et al., Nature 480, 336 (2011).

27. T. Zhou et al., Science 329, 811 (2010).

28. N. A. Doria-Rose et al., J. Virol. 84, 1631 (2010).

29. Y. Li et al., Nat. Med. 13, 1032 (2007).

30. Y. Li et al., J. Virol. 83, 1045 (2009).

31. J. M. Binley et al., J. Virol. 82, 11651 (2008).

32. E. S. Gray et al., J. Virol. 83, 8925 (2009).

33. L. M. Walker et al., PLoS Pathog. 6, e1001028 (2010).

34. A. K. Dhillon et al., J. Virol. 81, 6548 (2007).

35. P. D. Kwong, J. R. Mascola, Immunity 37, 412 (2012).

36. The 10 clusters included 9 epitope-specific clusters for the monoclonal antibodies and 1 cluster for CD4. Antibody clustering was not solely a function of neutralization breadth: Antibodies within a given cluster could have substantially different neutralization breadth, whereas antibodies in different clusters could have similar neutralization breadth (table S2).

37. A. Trkola et al., J. Virol. 70, 1100 (1996).

38. T. Zhou et al., Nature 445, 732 (2007). 
39. Z. Zhu et al., J. Virol. 85, 11401 (2011).

40. R. Diskin et al., Science 334, 1289 (2011).

41. Materials and methods are available as supplementary materials on Science Online.

42. One of the features of our approach is its ability to distinguish between antibodies targeting overlapping epitopes in a substantially different ways: There is a significant difference in the correlation coefficients for antibodies targeting a similar epitope versus the correlation coefficients for antibodies targeting different epitopes on the same site of vulnerability. Similarly, there is a significant difference for similar epitopes versus different sites of vulnerability; however, there is no significant difference for different epitopes on the same site of vulnerability versus different sites of vulnerability (fig. S1).

43. R. M. Cardoso et al., Immunity 22, 163 (2005).

44. Residue numbering throughout the paper is relative to strain HXB2, unless stated otherwise.

45. K. J. Doores et al., Proc. Natl. Acad. Sci. U.S.A. 107 17107 (2010).

46. Y. Li et al., J. Virol. 86, 11231 (2012).

47. To resolve the discrepancy between epitope mapping and neutralization-based predictions, we determined the cocrystal structure of VRCO6 in complex with the gp120 core and compared it with the structure of the VRC01-like antibody VRC03: The antibody variable-domain root mean square deviation upon alignment of the respective VRC06- and VRC03-bound gp120 cores was $1.45 \AA$ confirming the similarity in the modes of gp120 recognition by these two antibodies (Fig. $2 \mathrm{~B}$ and table S3). Although VRCO3 and VRCO6 exhibit extraordinary similarity in their mode of recognition of gp120 when compared with VRC01, unlike VRC01, antibodies VRC03 and VRCO6 contain a long insertion in the heavy-chain framework 3 region that falls in the vicinity of the gp120 bridging sheet. In the crystallized complexes of these antibodies and monomeric gp120 core (which lacks the full V1/V2 region), the insertions do not make substantial contact with gp120. On the functional trimer spike, however, these insertions likely make additional contacts with the antigen, extending the antibody epitopes as compared with VRC01. The result is that VRCO3 and VRCO6 cluster in the periphery of the VRC01 cluster.

48. The division into monoclonal antibody clusters was based on the clustering results from the larger 34-strain panel (Fig. 2A), whereas serum delineation was performed on an available 21-strain panel (because of serum volume constraints, we used a 21-strain subset of the main panel). Although antibody clustering is sensitive to the size of the viral panel (appendix S1), predictions with smaller panels (such as the 21-strain panel used in the serum analysis) could nonetheless be sufficient (fig. S3) delineation of component-antibody specificities for the CAP256 sera was based on a smaller 13-strain panel (41).

49. A signal for PGT128-like antibodies in donor 45 was also observed. However, only part of the neutralization activity of the donor 45 serum could be attributed to VRC01-like antibodies (fig. S11), which indicated that other antibody specificities may also exist in that serum. It is thus not surprising to observe other neutralization signals in the predictions.

50. P. L. Moore et al., J. Virol. 85, 3128 (2011)

51. L. M. Walker et al., Proc. Natl. Acad. Sci. U.S.A. 108, 20125 (2011).

52. Sera were from clade B-infected donors from the cohort described in (28) with moderate to broad neutralization on the panel of 21 viruses.

53. T. Zhou et al., Retrovirology 9 (Suppl. 2), P57 (2012).

54. For example, standard serum mapping for glycan-V3 antibodies uses N332 mutants and, thus, may not be as sensitive for some antibodies in the PGT128-like group that are more affected by glycan-301 (table S4).

55. The neutralization-based delineation showed relatively strong signals ( $>0.2$ ) for 2G12- or b12-like antibodies with many of the sera, and potential VRC01-, PG9PGT128-, and 10E8-like specificities were predicted for specific subsets of the sera. In the case of 2G12-like activity, many serum samples were also found to be positive by experimental mapping assays, which indicated that this epitope might be a more frequent target compared with other major epitopes, although not necessarily by antibodies having the specific domain-exchanged nature of 2G12 (Fig. 3D). A clear threshold for the presence or absence of a particular specificity in a given serum was not apparent: A level of 0.35 positively identified 10E8-like antibodies in donor N152, but sporadic signals of up to $\sim 0.3$ were observed in longitudinal samples (Fig. 3B).

56. To confirm the predicted similarity to VRCO1, we crystallized and determined the structure of the antigen-binding fragment (Fab) of VRC23 in complex with an HIV-1 core gp120. The epitope footprints on gp120 for VRC23 and VRC01 were similar, with buried gp120 surface areas correlating significantly (Spearman $R=0.76, P<0.0001$ ) (Fig. $4 \mathrm{~A}$ and figs. S8 to S10).

57. M. D. Simek et al., J. Virol. 83, 7337 (2009).

58. The current analysis is validated primarily for a single major component-antibody specificity in serum, with predictions on other specificities yet to be ascertained; however, the ability to extract major specificities from polyclonal sera will likely be of considerable utility. Nevertheless, the observation that antibody neutralization fingerprints can capture sufficient information to allow, at least in some cases, the deconvolution of the polyclonal serum-based pattern of HIV-1 neutralization into neutralization from component antibodies is subject to a number of assumptions and limitations (41)

59. J. M. Binley et al., J. Virol. 78, 13232 (2004).

60. M. S. Seaman et al., J. Virol. 84, 1439 (2010).

61. Q. J. Sattentau, A. J. McMichael, F1000 Biol. Rep. 2, 60 (2010).

62. F. Klein et al., J. Exp. Med. 209, 1469 (2012)

Acknowledgments: Support for this work was provided by the Intramural Research Program of the Vaccine Research Center, NIAID, NIH, and by the International AIDS Vaccine Initiative. The CAPRISA cohort was established through grants from NIAID, NIH (U19 Al51794) and the South African Department of Science and Technology. The authors are grateful to ]. Stuckey for assistance with graphics and thank B. Korber, D. Montefiori, and M. Roederer and members of the Structural Biology Section, Structural Bioinformatics Core, Humoral Immunology Section, and Humoral Immunology Core at the NIH Vaccine Research Center for helpful discussions or comments on the manuscript. We thank ]. Baalwa, D. Ellenberger, D. Gabuzda, F. Gao, B. Hahn, K. Hong, J. Kim, F. McCutchan, D. Montefiori, L. Morris, ]. Overbaugh, E. Sanders-Buell, G. Shaw, R. Swanstrom, M. Thomson, S. Tovanabutra, C. Williamson, and L. Zhang for contributing the HIV-1 Envelope plasmids used in our neutralization panels. The data presented in this manuscript are tabulated in the main paper and in the supplementary materials. The NIH has filed a patent relating to neutralizing antibodies in this manuscript: WIPO no. WO 2011/038290 A2, titled "Neutralizing antibodies to HIV-1 and their use," with inventors ].R.M., P.D.K., M.C., L.S., and G.O. Structure factors and coordinates for the VRCO6 and VRC23 antibody cocrystal structures with HIV-1 gp120 have been deposited with the Protein Data Bank under accession codes 4]B9 and 4]6R, respectively. Scripts for the neutralization-based antibody clustering and serologic analysis are included in the online supplementary material and methods (appendix S2) and are available from I.S.G or P.D.K. upon request. Use of sector 22 (Southeast Region Collaborative Access team) at the Advanced Photon Source was supported by the U.S. Department of Energy, Basic Energy Sciences, Office of Science, under contract no. W-31-109-Eng-38.

Supplementary Materials

www.sciencemag.org/cgi/content/full/340/6133/751/DC1 Materials and Methods

Supplementary Text

Figs. S1 to S15

Tables S1 to S10

References (63-84)

12 December 2012; accepted 28 March 2013

$10.1126 /$ science. 1233989

\section{Emergence of Individuality in Genetically Identical Mice}

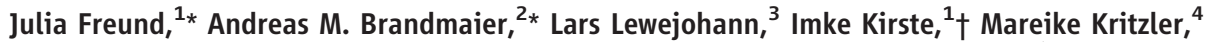
Antonio Krüger, ${ }^{4,5}$ Norbert Sachser, ${ }^{3}$ Ulman Lindenberger, ${ }^{2}$ Gerd Kempermann ${ }^{1,6} \ddagger$

Brain plasticity as a neurobiological reflection of individuality is difficult to capture in animal models. Inspired by behavioral-genetic investigations of human monozygotic twins reared together, we obtained dense longitudinal activity data on 40 inbred mice living in one large enriched environment. The exploratory activity of the mice diverged over time, resulting in increasing individual differences with advancing age. Individual differences in cumulative roaming entropy, indicating the active coverage of territory, correlated positively with individual differences in adult hippocampal neurogenesis. Our results show that factors unfolding or emerging during development contribute to individual differences in structural brain plasticity and behavior. The paradigm introduced here serves as an animal model for identifying mechanisms of plasticity underlying nonshared environmental contributions to individual differences in behavior.

$\mathrm{P}$ lasticity, or the reciprocal interaction between brain structure and function, draws on genetic and nongenetic sources of variation and forms the neurobiological basis of individuality. Behavioral-genetic studies with humans provide statistical tools for estimating the additive and interactive contributions of genetic and environmental variations to individual differences in behavioral development (1). In the case of monozygotic twins reared together, sibling differences reflect the influence of individual responses, based on the same genetic makeup, to a nominally identical environment. Somewhat paradoxically, this source of variation is generally referred to as the "nonshared environment" in behavior genetics. As Turkheimer has noted, "exactly what the nonshared environment consists of has been a matter of mystery and controversy for some time" [(2) p. 826]. We developed an animal model for studying the nonshared environment and examined its effects on behavioral and neural development.

In rodents, enriched environments are among the tools of choice for addressing the influence of a given environment on individuals with identical genetic background $(3,4)$. However, with some exceptions $[(5)$, see also $(3,6)]$ the emergence of experience-based individual differences within groups of genetically identical animals exposed 


\section{Science MIAAAS}

\section{Delineating Antibody Recognition in Polyclonal Sera from} Patterns of HIV-1 Isolate Neutralization

Ivelin S. Georgiev, Nicole A. Doria-Rose, Tongqing Zhou, Young Do Kwon, Ryan P. Staupe, Stephanie Moquin, Gwo-Yu Chuang, Mark K. Louder, Stephen D. Schmidt, Han R. Altae-Tran, Robert T. Bailer, Krisha McKee, Martha Nason, Sijy O'Dell, Gilad Ofek, Marie Pancera, Sanjay Srivatsan, Lawrence Shapiro, Mark Connors, Stephen A. Migueles, Lynn Morris, Yoshiaki Nishimura, Malcolm A. Martin, John R. Mascola and Peter D. Kwong (May 9, 2013) Science 340 (6133), 751-756. [doi: 10.1126/science.1233989]

Editor's Summary

\section{Building Better Vaccines}

In the past few years, several highly potent, broadly neutralizing antibodies (bNAbs) specific for the gp120 envelope protein of HIV-1 have been discovered. The goal of this work is to use this information to inform the design of vaccines that are able to induce such antibodies (see the Perspective by Crowe). However, because of extensive somatic hypermutation, the epitope bound by these antibodies often does not bind to the germline sequence. Jardine et al. (p. 711, published online 28 March; see the cover) used computational analysis and in vitro screening to design an immunogen that could bind to VRC01-class bNAbs and to their germline precursors. Georgiev et al. (p. 751) took advantage of the fact that only four sites on the HIV viral envelope protein seem to bind bNAbs, and sera that contain particular bNAbs show characteristic patterns of neutralization. An algorithm was developed that could successfully delineate the neutralization specificity of antibodies present in polyclonal sera from HIV-infected patients.

This copy is for your personal, non-commercial use only.

Article Tools

Permissions
Visit the online version of this article to access the personalization and article tools:

http://science.sciencemag.org/content/340/6133/751

Obtain information about reproducing this article: http://www.sciencemag.org/about/permissions.dtl

Science (print ISSN 0036-8075; online ISSN 1095-9203) is published weekly, except the last week in December, by the American Association for the Advancement of Science, 1200 New York Avenue NW, Washington, DC 20005. Copyright 2016 by the American Association for the Advancement of Science; all rights reserved. The title Science is a registered trademark of AAAS. 